\title{
THROUGHPUT MAXIMIZING FEEDBACK FOR MIMO OFDM BASED WIRELESS COMMUNICATION SYSTEMS
}

\author{
Stefan Schwarz and Markus Rupp \\ Institute of Telecommunications, Vienna University of Technology \\ Gusshausstrasse 25/389, A-1040 Vienna, Austria \\ Email: $\{$ sschwarz, mrupp $\} @$ nt.tuwien.ac.at
}

\begin{abstract}
Currently evolving wireless communication systems (e.g. LTE-A, WiMAX) are based on linearly precoded MIMO OFDM and utilize adaptive modulation and coding to adjust the code rate and modulation alphabet to the current channel conditions. For this purpose the transmitter requires channel knowledge, which is provided by means of receiver feedback, utilizing the rank, precoding matrix and channel quality indicators. This work presents algorithms for computing these feedback values. Our algorithms choose the rank and precoding matrix indicators to maximize the estimated user throughput, whereas the channel quality indicator is chosen more conservatively to satisfy a given upper bound on the block error ratio. We demonstrate the performance of the proposed algorithms by means of link level simulations and comparison to theoretical bounds on the achievable throughput.
\end{abstract}

Index Terms - LTE, WiMAX, Link Adaptation, Feedback, Precoding, MIMO

\section{INTRODUCTION}

Multiple Input Multiple Output (MIMO) Orthogonal Frequency Division Multiplexing (OFDM) is the basis of practically all modern standardized high performance wireless communication systems, e.g. 3GPP's Long Term Evolution Advanced (LTE-A) and IEEE's Worldwide Interoperability for Microwave Access 2 (WiMAX 2). Furthermore these systems employ Bit Interleaved Coded Modulation (BICM) to adapt the code rate and modulation alphabet to the current channel conditions. To increase the spectral efficiency, code book based unitary precoding is supported. Because the transmitter does not have the necessary channel knowledge to exploit these features, feedback from the receiver is required. For this purpose three feedback values are typically utilized. The Rank Indicator (RI) and Precoding Matrix Indicators (PMIs) are employed for MIMO pre-processing. They choose the appropriate transmission rank and precoders, to achieve high spectral efficiency. Additionally, the Channel Quality Indicator (CQI) is employed to signal the supported Adaptive Modulation and Coding (AMC) scheme to achieve

This work has been funded by Deutsche Telekom AG and the Institute of Telecommunications, Vienna University of Technology.

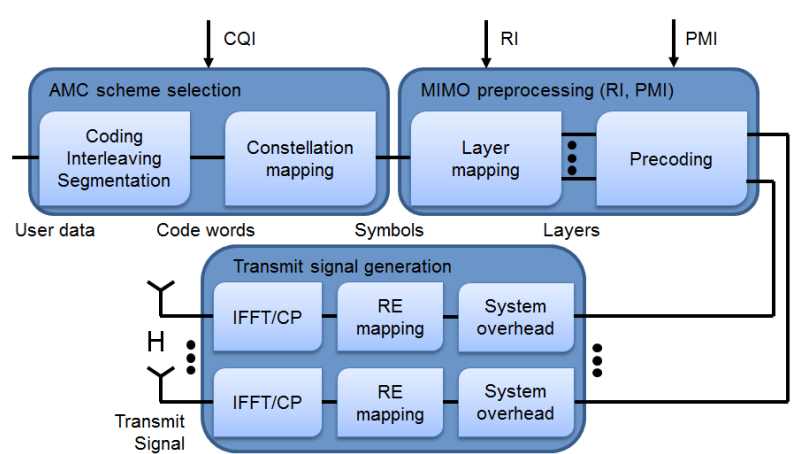

Fig. 1. System architecture of a modern wireless communication system.

a target Block Error Ratio (BLER), given the current channel conditions. Figure 1 shows a schematic of the considered system architecture as it is employed in LTE-A and WiMAX 2.

In order to maximize the throughput of a user, sophisticated algorithms to compute the feedback indicators are required. We propose algorithms that utilize Mutual Information Effective SNR Mapping (MIESM) [1] to estimate and maximize the achievable throughput of a user. This work is based on results we previously obtained for Long Term Evolution (LTE) [2]. We generalize and extend this work and improve the estimators, especially for large antenna configurations as utilized in LTE-A (up to $8 \times 8$ ) and WiMAX 2 (up to $12 \times 12$ ).

In Section 2, we introduce the employed system model. Next, in Section 3, we derive the optimization problem for estimating the User Equipment (UE) feedback indicators. Due to the high complexity of the solution of this optimization problem, we derive simplified sequential algorithms in Section 4. Afterwards, we apply the proposed sequential algorithms to an LTE-A compliant system by means of link level simulations, utilizing the Vienna LTE Link Level Simulator [3]. Furthermore, in Section 5, we compare the performance in terms of achieved throughput to theoretical throughput bounds derived in [4]. We partly adopt LTE notation as we mainly focus on that system.

\section{SYSTEM MODEL}

We consider linearly precoded MIMO OFDM systems utilizing BICM, as shown in Figure 1. OFDM divides the total 
system bandwidth into $K$ orthogonal subcarriers. Furthermore, a temporal frame structure is superimposed onto the individual OFDM symbols for signaling purposes. In LTE 14 OFDM symbols are combined to a subframe of $1 \mathrm{~ms} \mathrm{du}-$ ration. In that way a time-frequency grid of OFDM samples or Resource Elements (REs) is spanned onto which the user data is mapped. The number of REs per subframe is denoted as $R$ and $r$ indicates a specific RE. Multiple access is handled by means of Orthogonal Frequency Division Multiple Access (OFDMA), separating individual users by assigning orthogonal REs.

In order to adapt the transmission parameters to the current channel conditions, the transmitter requires Channel State Information (CSI). This is provided by means of the CQI, PMI and RI feedback from the receiver.

In a MIMO system multiple parallel data streams (layers) can be transmitted. In general each layer has different channel quality. It follows that the supported AMC scheme on each layer is different as well. For that purpose multiple code words are employed to allow different AMC schemes on the individual layers. In LTE-A the number of code words is restricted to two, whereas the number of spatial layers can be up to eight, requiring one code word to be mapped onto multiple layers. The set of layers belonging to a code word $c$ is denoted $\mathcal{L}_{c}$. The supported AMC scheme is signaled by means of the CQI. Therefore individual CQIs are required for each code word. Furthermore, the system architecture of Figure 1 implies that the same AMC scheme is utilized on all of the assigned resources. Nevertheless, it can be beneficial to provide time-frequency selective CQI feedback in order to allow for sophisticated multiuser scheduling [5]. For that purpose the total of $R$ resources is divided into $S$ subbands and CQI feedback is provided for each subband $s \in\{1, \ldots, S\}$. The set of REs belonging to subband $s$ is denoted $\mathcal{R}_{s}$. We define a surjective mapping $\rho:\{1, \ldots, R\} \rightarrow\{1, \ldots, S\}$ assigning each RE $r$ to the corresponding subband $s$. In LTE the minimum size of a subband is equal to a Resource Block (RB) consisting of 12 subcarriers and 14 OFDM symbols.

The preferred number of spatial layers $L$ (called rank) is signaled by means of the RI. The same rank is employed on all assigned resources. In order to exploit the potential MIMO gains, MIMO preprocessing of the data symbols is required. LTE restricts this MIMO preprocessing to multiplication of the data symbols with a unitary precoding matrix, chosen from a finite code book. The PMIs are utilized to signal the favored precoders. Depending on the setup of the considered communication system, different precoders can be employed on different subbands or one single wideband precoder is applied on all resources. We catch both possibilities by defining the precoder for subband $s$, denoted $\mathbf{W}_{s}$, as the product of a wideband precoder $\mathbf{W}^{(1)}$ and a subband precoder $\mathbf{W}_{s}^{(2)}$ :

$$
\mathbf{W}_{s}=\mathbf{W}^{(1)} \mathbf{W}_{s}^{(2)} \text {. }
$$

This notation is especially suitable for the eight transmit an- tenna code book of LTE-A. Here, a precoder is indicated with a two value index. As shown in the 3GPP RAN document [6] this code book can equivalently be written as the product of a wideband and a subband precoder, each stemming from individual, rank dependent code books $\mathcal{W}_{1}^{(L)}$ and $\mathcal{W}_{2}^{(L)}$. Otherwise, we identify one of the two code books with $\{1\}$, depending on whether wideband or subband precoder feedback is considered.

Assuming $N_{t}$ transmit and $N_{r}$ receive antennas, the inputoutput relation on RE $r$ is given by:

$$
\mathbf{y}_{r}=\mathbf{H}_{r} \mathbf{W}_{s} \mathbf{x}_{r}+\mathbf{n}_{r}, \quad r \in\{1, \ldots, R\}, \quad s=\rho(r) .
$$

Here, $\mathbf{H}_{r} \in \mathbb{C}^{N_{r} \times N_{t}}$ denotes the channel matrix. The transmit symbol vector is denoted $\mathbf{x}_{r} \in a^{L \times 1}$ and $\mathbf{n}_{r} \sim$ $\mathcal{C N}\left(0, \sigma_{n}^{2} \cdot \mathbf{I}_{N_{r}}\right)$ refers to the white Gaussian receiver noise. Furthermore, $a$ denotes one of the supported modulation alphabets $\mathcal{A}$ (e.g. 4/16/64 QAM). For the transmission rank $L$ holds the inequality $L \leq L_{\max }=\operatorname{rank}\left(\mathbf{H}_{r}\right)$.

With a linear equalizer filter $\mathbf{F}_{r} \in \mathbb{C}^{L \times N_{r}}$ at the receiver, the post-equalization symbol vector $\mathbf{r}_{r} \in \mathbb{C}^{L \times 1}$ is given by:

$$
\mathbf{r}_{r}=\mathbf{F}_{r} \mathbf{y}_{r}=\underbrace{\mathbf{F}_{r} \mathbf{H}_{r} \mathbf{W}_{s}}_{\mathbf{K}_{r}} \mathbf{x}_{r}+\mathbf{F}_{r} \mathbf{n}_{r} .
$$

Then the post-equalization Signal to Interference and Noise Ratio (SINR) for RE $r$ and transmission layer $l$ equals:

$$
\operatorname{SINR}_{r, l}\left(\mathbf{W}_{s}\right)=\frac{P_{l} \cdot\left|\mathbf{K}_{r}[l, l]\right|^{2}}{\sum_{i \neq l} P_{i} \cdot\left|\mathbf{K}_{r}[l, i]\right|^{2}+\sigma_{n}^{2} \sum_{i}\left|\mathbf{F}_{r}[l, i]\right|^{2}},
$$

Here, $\mathbf{K}_{r}[l, i]$ denotes the element in the $l$ th row and $i$ th column of the matrix $\mathbf{K}_{r}$ and $P_{l}$ refers to the transmit power on layer $l$. The SINR depends on the utilized precoder and, via the precoder, on the number of transmission layers $L$. Our proposed algorithms are restricted to receivers for which a post-equalization SINR can be stated.

\section{COMPUTATION OF FEEDBACK INDICATORS}

Our goal for the choice of the feedback values is to maximize the estimated spectral efficiency of a user. Additionally the CQI is chosen to satisfy an upper bound on the BLER.

To estimate the BLER of each AMC scheme $m \in \mathcal{M}$, for a given rank $L$ and precoders $\left\{\mathbf{W}_{s}\right\}_{s=1}^{S}$, MIESM [1] is employed to obtain an average Signal to Noise Ratio (SNR) for the considered REs. MIESM utilizes the BICM capacity $f_{m}(\mathrm{SNR})^{1}$, corresponding to the modulation alphabet of AMC scheme $m$, to average the SINRs experienced on the considered REs [7]. The average SNR corresponds to an AWGN channel that achieves the same spectral efficiency as the fading channel does, over the considered resources. Then, we utilize an AWGN SNR to BLER mapping table $g_{m}(\mathrm{SNR})$ to obtain the estimated BLER of each AMC scheme $m$, denoted $P_{m}$. Furthermore, we define a function $h_{m}\left(P_{m}\right)$ that

\footnotetext{
${ }^{1}$ The calibration for each AMC scheme is already included in $f_{m}$
} 
delivers the spectral efficiency $e_{m}$ for AMC scheme $m$, if the BLER target $P_{b}^{(t)}$ is satisfied and zero otherwise:

$$
h_{m}(P)=\left\{\begin{array}{c}
e_{m}, P_{m} \leq P_{b}^{(t)} \\
0, P_{m}>P_{b}^{(t)}
\end{array} .\right.
$$

For the mathematical formulation of the optimization problem we need to distinguish between the cases of wideband and subband specific CQI feedback. In the subband specific CQI case, MIESM averaging is applied for each subband, delivering the subband $\mathrm{SNR}_{s}^{c}$ for code word $c$ :

$\operatorname{SNR}_{s}^{c}\left(\mathbf{W}_{s}, m\right)=f_{m}^{-1}\left(\frac{1}{\left|\mathcal{R}_{s}\right|\left|\mathcal{L}_{c}\right|} \sum_{r \in \mathcal{R}_{s}, l \in \mathcal{L}_{c}} f_{m}\left(\operatorname{SINR}_{r, l}\left(\mathbf{W}_{s}\right)\right)\right)$

Here, $|\cdot|$ denotes the cardinality of the set and $f_{m}^{-1}(\cdot)$ refers to the inverse of $f_{m}(\cdot)$. Via the precoder, this average SNR also depends on the transmission rank $L$. The corresponding estimated BLER equals:

$$
P_{s}^{c}\left(\mathbf{W}_{s}, m\right)=g_{m}\left(\operatorname{SNR}_{s}^{c}\left(\mathbf{W}_{s}, m\right)\right) .
$$

This delivers the estimated spectral efficiency for subband $s$ and code word $c$ :

$$
E_{s}^{c}\left(\mathbf{W}_{s}, m\right)=h_{m}\left(P_{s}^{c}\left(\mathbf{W}_{s}, m\right)\right) \cdot\left(1-P_{s}^{c}\left(\mathbf{W}_{s}, m\right)\right) .
$$

With this, we are ready to formulate the subband optimization problem for the feedback indicators:

$$
\begin{aligned}
& {\left[\hat{L},\left\{\hat{\mathbf{W}}_{s}\right\}_{S},\left\{\hat{\mathbf{m}}_{s}\right\}_{S}\right] \underset{L, \mathbf{W}^{(1)}, \mathbf{W}_{s}^{(2)}, \mathbf{m}_{s}}{\operatorname{argmax}} \sum_{s=1}^{S} \sum_{c=1}^{C_{L}} E_{s}^{c}\left(\mathbf{W}_{s}, \mathbf{m}_{s}[c]\right)} \\
& \text { subject to: } L \leq L_{\max } \\
& \mathbf{W}^{(1)} \in \mathcal{W}_{1}^{(L)} \\
& \mathbf{W}_{s}^{(2)} \in \mathcal{W}_{2}^{(L)} \\
& \mathbf{m}_{s} \in \mathcal{M}^{C_{L} \times 1}
\end{aligned}
$$

Here, $C_{L}$ denotes the rank dependent number of code words. The preferred RI is given by $\hat{L}$, the PMIs correspond to the indices of the $S$ precoders $\left\{\hat{\mathbf{W}}_{s}\right\}_{S}$ and the CQIs for each code word and subband follow from the favored AMC schemes $\left\{\hat{\mathbf{m}}_{s}\right\}_{S}$. Note that the optimization with respect to the subband precoders $\mathbf{W}_{s}^{(2)}$ and subband AMCs $\mathbf{m}_{s}$ is independent for distinct subbands, given the rank and wideband precoder choice. It can therefore be split into individual subband problems for each choice of $L$ and $\mathbf{W}^{(1)}$.

For wideband specific CQI feedback, an average code word dependent $\mathrm{SNR}^{c}$ needs to be computed for all $R$ resources:

$$
\begin{aligned}
& \operatorname{SNR}^{c}\left(\left\{\mathbf{W}_{s}\right\}_{S}, m\right)= \\
& f_{m}^{-1}\left(\frac{1}{R\left|\mathcal{L}_{c}\right|} \sum_{r=1}^{R} \sum_{l \in \mathcal{L}_{c}} f_{m}\left(\operatorname{SINR}_{r, l}\left(\mathbf{W}_{s}\right)\right)\right), s=\rho(r) .
\end{aligned}
$$

That SNR is then mapped to a code word dependent estimated spectral efficiency for all resources:

$$
\begin{aligned}
& P^{c}\left(\left\{\mathbf{W}_{s}\right\}_{S}, m\right)=g_{m}\left(\operatorname{SNR}^{c}\left(\left\{\mathbf{W}_{s}\right\}_{S}, m\right)\right), \\
& E^{c}\left(\left\{\mathbf{W}_{s}\right\}_{S}, m\right)=h_{m}\left(P^{c}\right) \cdot\left(1-P^{c}\right) .
\end{aligned}
$$

The wideband optimization problem is then formulated as:

$$
\left[\hat{L},\left\{\hat{\mathbf{W}}_{s}\right\}_{S}, \hat{\mathbf{m}}\right] \underset{L, \mathbf{W}^{(1)},\left\{\mathbf{W}_{s}^{(2)}\right\}_{S}, \mathbf{m}}{\operatorname{argmax}} \sum_{c=1}^{C_{L}} E_{s}^{c}\left(\left\{\mathbf{W}_{s}\right\}_{S}, \mathbf{m}[c]\right)
$$
subject to: $L \leq L_{\max }$

$$
\begin{aligned}
& \mathbf{W}^{(1)} \in \mathcal{W}_{1}^{(L)} \\
& \mathbf{W}_{s}^{(2)} \in \mathcal{W}_{2}^{(L)} \\
& \mathbf{m} \in \mathcal{M}^{C_{L} \times 1}
\end{aligned}
$$

Solving these optimization problems implies very high computational complexity, because they are combinatorial problems, that can in general only be solved by an exhaustive search $^{2}$. We therefore propose a simplified, suboptimal algorithm in the following section.

\section{APPROXIMATE SEQUENTIAL SOLUTION}

To reduce the complexity of the optimization problems described in the previous section, we resort to a sequential optimization as suggested in [8]. In this approach, the precoders and transmission rank are chosen in advance and afterwards, with knowledge of these values, the optimal CQIs are selected by solving (9) or (13).

The choice of the precoders is based on maximizing the sum spectral efficiency, estimated by means of the BICM capacity. For this purpose we first need to compute the spectral efficiency of RE $r$, denoted $I_{r}$, for each combination of precoders and transmission ranks. Because the BICM capacity is modulation alphabet dependent, we utilize a function $f(\mathrm{SNR})$ that delivers the maximum efficiency over all modulation alphabets. This step is necessary to allow an independent choice of the subband precoders of distinct subbands.

$$
I_{r}\left(\mathbf{W}_{s}, L\right)=\sum_{c=1}^{C_{L}} \sum_{l \in \mathcal{L}_{c}} f\left(\operatorname{SINR}_{r, l}\left(\mathbf{W}_{s}\right)\right)
$$

The most expensive step is the computation of the postequalization SINR. The spectral efficiency is obtained with a simple table look up. To choose the subband precoder $\mathbf{W}_{s}^{(2)}$, we maximize the sum spectral efficiency for each subband:

$$
\begin{array}{r}
I_{s}\left(\mathbf{W}_{s}, L\right)=\sum_{r \in \mathcal{R}_{s}} I_{r}\left(\mathbf{W}_{s}, L\right), \\
\hat{\mathbf{W}}_{s}^{(2)}\left(\mathbf{W}^{(1)}, L\right)=\underset{\mathbf{W}_{s}^{(2)} \in \mathcal{W}_{2}^{(L)}}{\operatorname{argmax}} I_{s}\left(\mathbf{W}_{s}, L\right) .
\end{array}
$$

${ }^{2}$ Already for an LTE-A system with $N_{t} \times N_{r}=8 \times 1$ antennas and 1.4 MHz bandwidth assuming RB selective PMI feedback and wideband CQI feedback the number of possible combinations is equal to $4 \cdot 10^{9}$. 
Table 1. Collection of the main simulation parameters.

\begin{tabular}{lc}
\hline Channel model & VehA [9] \\
Bandwidth & $1.4 \mathrm{MHz}$ \\
Receiver & Zero forcing \\
Antenna correlation & 0 \\
Feedback delay & 0 \\
Subband size & $1 \mathrm{RB}$ \\
Channel knowledge & Perfect \\
\hline
\end{tabular}

Utilizing this pre-knowledge of the subband precoders, it is possible to obtain the corresponding optimal wideband precoder, rank and CQIs from (9) or (13).

Alternatively, the optimization problem can further be simplified by choosing the wideband precoder in advance as well. Then, the wideband precoder $\hat{\mathbf{W}}^{(1)}$ follows from the sum spectral efficiency over all subbands:

$$
\begin{array}{r}
I\left(\mathbf{W}_{s}, L\right)=\sum_{s=1}^{S} I_{s}\left(\mathbf{W}_{s}, L\right), \\
\hat{\mathbf{W}}^{(1)}(L)=\underset{\mathbf{W}^{(1)} \in \mathcal{W}_{1}^{(L)}}{\operatorname{argmax}} I\left(\mathbf{W}_{s}, L\right) .
\end{array}
$$

Plugging this precoder back into (16) we obtain the corresponding, rank dependent subband precoders $\hat{\mathbf{W}}_{s}^{(2)}(L)=$ $\hat{\mathbf{W}}_{s}^{(2)}\left(\hat{\mathbf{W}}^{(1)}(L), L\right)$. Utilizing this pre-knowledge of the wideband and subband precoders, the optimization problems (9) and (13) are further simplified. One can even go a step further to choose the rank in advance as well:

$$
\hat{L}=\underset{L \leq L_{\max }}{\operatorname{argmax}} I\left(\hat{\mathbf{W}}_{s}(L), L\right), \hat{\mathbf{W}}_{s}(L)=\hat{\mathbf{W}}^{(1)}(L) \hat{\mathbf{W}}_{s}^{(2)}(L) .
$$

Then the only variables left for optimization in (9) and (13) are the $\mathrm{CQIs}{ }^{3}$.

We decided not to simplify the computation of the CQIs, because we observed that they are more performance critical than the RI and the PMIs. Choosing the wrong CQI either under estimates the possible performance, leading to a waste of resources, or in case of over estimated performance the BLER bound will not be satisfied anymore.

We recognized, during our simulations of an LTE-A system, that the performance degradation with each of the simplifying steps described above increases with the number of transmission layers. In a system with $N_{t} \times N_{r}=8 \times 2$ antennas, there is no performance difference between choosing just the subband precoders in advance or choosing the subband and wideband precoders and additionally the rank in advance. On the other in an $8 \times 8$ system, each of the simplifying steps leads to a noticeable performance degradation.

Further simplifications are possible by exploiting time and frequency correlation. To reduce the amount of SINR computations, we sub-sample the resource grid. In our simulations,

\footnotetext{
${ }^{3}$ For the same example as before these three steps reduce the number of possible choices to $23 \cdot 10^{3}$.
}

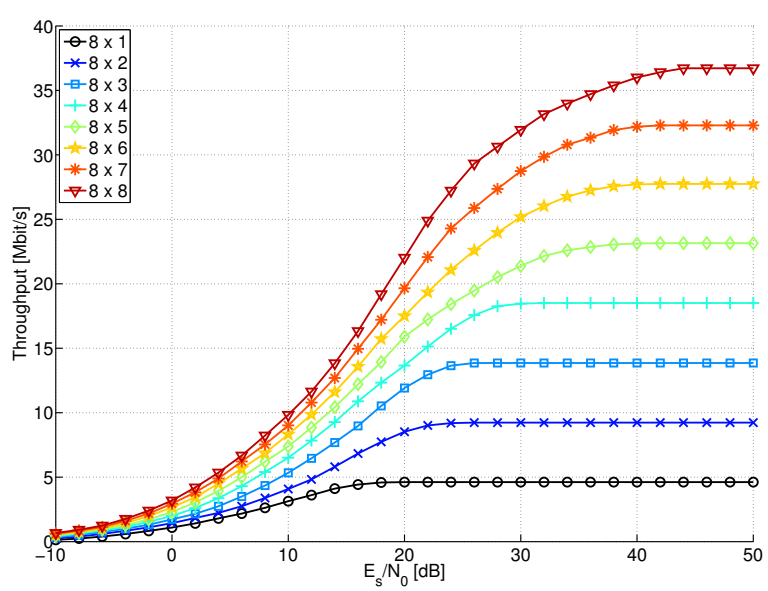

Fig. 2. Comparison of the throughput for different LTE-A antenna configurations $N_{t} \times N_{r}$.

utilizing a VehA [9] channel model with coherence bandwidth of $1.35 \mathrm{MHz}$, we compute post-equalization SINRs just on every third subcarrier without degrading the performance. Furthermore, we do not consider all possible ranks in the optimization, but, utilizing temporal correlation, optimize only over the set $\{\mathrm{RI}-1, \mathrm{RI}, \mathrm{RI}+1\}$ with RI being the previously reported rank, without degrading the performance. Further investigations in this respect are required.

\section{SIMULATION RESULTS}

In this section, we present simulation results acquired with the (LTE-A extended) Vienna LTE Link Level Simulator [3]. In previous papers, concerned with LTE feedback, we compared the throughput obtained with our feedback estimators to the optimum throughput, determined by exhaustive search [2]. Unfortunately, finding the optimum throughput in LTEA is computationally not feasible anymore. Therefore, we compare the performance utilizing our estimators to theoretical throughput bounds derived in [4]. In [4] we also derived a low complexity method to predict the optimum throughput, that proved to be accurate for LTE. We adopt this method here as well, although we cannot assure that the prediction is accurate in LTE-A too. The prediction of the optimum performance is based on link level abstraction methods and an exhaustive search over all precoder combinations.

In the presented simulations we employ the sequential optimization algorithm described in Section 4. We select the subband and wideband precoders in advance but not the rank. We furthermore utilize wideband CQI feedback. The main simulation parameters are summarized in Table 1. The simulation results are averaged over 1000 subframes, giving 1000 independent channel and noise realizations. We compute feedback values for each subframe and assume a feedback delay of zero, meaning that the feedback is known before data transmission.We choose this setting to investigate the ideal performance of the feedback method without influence of outdated feedback. The receiver has perfect channel knowledge. 


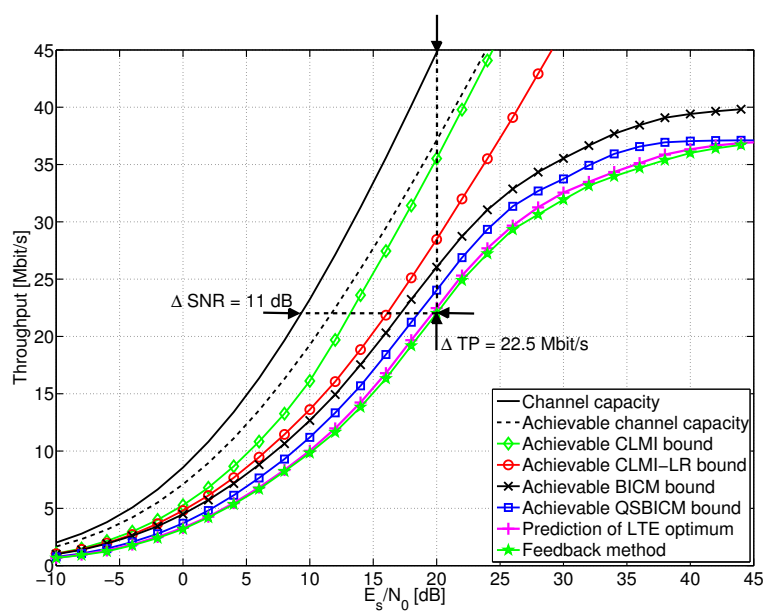

Fig. 3. Comparison of the throughput of an $N_{t} \times N_{r}=8 \times 8$ system to theoretical throughput bounds.

Figure 2 shows the throughput obtained with different antenna configurations $N_{t} \times N_{r}$ over the average receive SNR (ratio of symbol energy and noise power spectral density). It can be observed that a very large SNR of more than $40 \mathrm{~dB}$ is required to fully exploit the throughput of the $8 \times 8$ system.

In Figure 3, we compare the throughput of an $N_{t} \times N_{r}=$ $8 \times 8$ system to the throughput bounds derived in [4]. The following observations can be made:

- Comparing the leftmost curve, channel capacity, to the rightmost curve, the performance of the simulated LTE-A system, at a typical operating point of $20 \mathrm{~dB}$, reveals a performance loss of approximately 50\%. A similar behavior was also observed for an LTE system in [4].

- Taking into account the system overhead, with the achievable channel capacity, causes a loss of $18 \%$ in throughput compared to channel capacity at SNR $=20 \mathrm{~dB}$. This already explains a major part of the total performance loss.

- If we take into account the finite set of possible precoders, defined in the LTE-A standard, and the fact that the transmit power is equally distributed over the subcarriers (instead of water filling power allocation), we can explain another loss of $2.5 \%$ in throughput (achievable CLMI bound). Note that this loss is much more severe at low SNR.

- The achievable CLMI-LR bound considers the utilization of the Zero Forcing equalizer, which causes a loss of $18 \%$.

- The considered practical system is based on BICM instead of Gaussian signaling. This causes a throughput loss of $2.5 \%$ of channel capacity (achievable BICM bound).

- Finally, the code block length and the set of supported AMC schemes are both finite. This is considered in the achievable QSBICM bound and explains a throughput loss of $2.5 \%$ of channel capacity.

The gap of $1 \mathrm{~dB}$ that is still left between the QSBICM bound and the simulated performance is due to the sub optimality of the employed channel codes and the feedback method.
The performance loss of the feedback method compared to the predicted optimum performance is in the order of $0.2-$ $1 \mathrm{~dB}$ depending on the considered throughput. A similar loss is also observed for an LTE system in [4].

\section{CONCLUSION}

In this work, we derive the optimization problem for computing optimal receiver feedback values for a MIMO BICM OFDM wireless communication system, that utilizes finite code book based precoding. Such a system requires CQI feedback, to employ the appropriate AMC scheme, and furthermore RI and PMI feedback for MIMO preprocessing. To avoid the high complexity involved in solving the derived optimization problem for obtaining these feedback indicators, we propose a simplified sequential solution. We present simulation results for an LTE-A system and investigate the gain of increasing the number of transmit antennas. Furthermore, we compare the performance of our proposed method to theoretical bounds, demonstrating its capabilities.

\section{REFERENCES}

[1] L. Wan, S. Tsai, and M. Almgren, "A Fading-Insensitve Performance Metric for a Unified Link Quality Model," in Proc. IEEE Wireless Communications \& Networking Conference WCNC, 2006.

[2] S. Schwarz, C. Mehlführer, and M. Rupp, "Calculation of the Spatial Preprocessing and Link Adaption Feedback for 3GPP UMTS/LTE," in Proc. IEEE Wireless Advanced 2010, London, UK, 2010.

[3] C. Mehlführer, J. C. Ikuno, M. Simko, S. Schwarz, and M. Rupp, "The Vienna LTE Simulators - Enabling Reproducibility in Wireless Communications Research," 2011, under review at EURASIP JASP.

[4] S. Schwarz, M. Simko, and M. Rupp, "On Performance Bounds for MIMO OFDM based Wireless Communication Systems," in Signal Processing Advances in Wireless Communications SPAWC 2011, San Francisco, CA, 2011.

[5] S. Schwarz, C. Mehlführer, and M. Rupp, "Low Complexity Approximate Maximum Throughput Scheduling for LTE," in 44th Annual Asilomar Conference on Signals, Systems, and Computers, Pacific Grove, California, Nov. 2010.

[6] Technical Specification Group RAN, "Way forward on 8Tx codebook for Rel.10 DL MIMO," Tech. Rep. R1 105011, 3GPP, Aug. 2010.

[7] G. Caire, G. Taricco, and E. Biglieri, "Capacity of bitinterleaved channels," Electron. Lett., vol. 32, issue 12, pp. 1060-1061, 1996.

[8] 3GPP, "E-UTRA and E-UTRAN; Physical layer procedures (Release 10)," December 2010, [Online]. http://www.3gpp.org/ftp/Specs/html-info/36213.htm.

[9] 3GPP, "Technical Specification Group Radio Access Networks; Deployment aspects (Release 8)," Dec. 2008, [Online]. http://www.3gpp.org/ftp/Specs/htmlinfo/25943.htm. 